\title{
A Modified Algorithmic Approach of DSDV Routing Protocol for Wireless Ad Hoc Network
}

\author{
Sourish Mitra ${ }^{1}$, Rafiqul Islam², Kanishka Mukherjee ${ }^{3}$, Abhishek Das $^{4}$, \\ Sohini Nandi ${ }^{5}$ \\ 1,2,3,4,5 CSE Dept, Guru Nanak Institute of Technology, Sodepur, Kolkata, W.B, India
}

\begin{abstract}
An ad-hoc network is the cooperative engagement of a collection of Mobile Hosts without the required intervention of any centralized Access Point. A Mobile Ad hoc NETwork called MANET is a kind of wireless ad-hoc network that is a self configuring network of mobile routers. These mobile routers are connected by wireless links. In MANET there are various routing protocols available. DSR, AODV and DSDV are most popular. Our proposed works are related to examine routing protocol for mobile ad hoc networks -the Destination Sequenced Distance Vector (DSDV) and On Demand protocol that evaluates both protocols based on the packet delivery fraction and average delay while varying number of sources and pause time. In this Improved -DSDV approach we can overcome the problem of state routes, as well as improve the performance of regular DSDV. We compare the performance of our work with DSDV. In our improved DSDV routing protocol, nodes can cooperate together to obtain an objective opinion about another nodes trustworthiness.
\end{abstract}

Keywords: Wireless communications, Broken node, Security, nodes, AODV, DSDV, Packet Delivery Fraction, MANET.

\section{INTRODUCTION}

A mobile ad-hoc network (MANET) is a self-configuring infrastructure less network of mobile devices connected by wireless. Ad hoc is Latin and means "for this purpose". Each device in a MANET is free to move independently in any direction, and will therefore change its links to other devices frequently. Each must forward traffic unrelated to its own use, and therefore be a router. The primary challenge in building a MANET is equipping each device to continuously maintain the information required to properly route traffic. Such networks may operate by themselves or may be connected to the larger Internet. MANETs are a kind of

wireless ad hoc networks that usually has a routable networking environment on top of a Link Layer ad hoc network. In the integrated MANET-Internet communication, a connection could be disrupted either by attacks on the Internet connectivity or by attacks on the ad hoc routing protocols. Because of this almost all possible attack on the traditional ad -

hoc networks also exist in the integrated wired and mobile ad hoc networks. The other factors which need to be considered

while choosing a protocol for MANETs are: Multicasting, Loop Free, Multiple routes, Distributed Operation, Unidirectional Link etc.

\section{Mobile Ad-Hoc Network Routing Protocols}

MANET Routing protocols are broadly classified into three types such as Proactive, Reactive and Hybrid protocols.

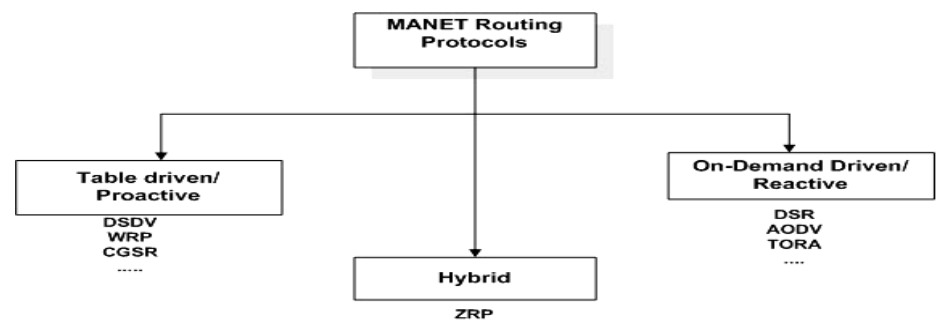

Proactive MANET protocols (PMPs) constantly update network topology information and ensure that it is available to all nodes. PMPs reduce network latency (or system time delay) but increase data overhead by constantly updating routing information. It ensures routes to all destinations are up-to-date and ready for use when required. Reactive MANET protocols determine routing paths only when required. They are associated with lower protocol overheads but longer packet delays. Examples of reactive and proactive protocols include AODV (reactive protocol) and OLSR (proactive protocol). Hybrid MANET routing protocols integrate suitable 
proactive and reactive MANET protocols. The resulting hybrid protocol achieves better performance than its components and is able to adjust dynamically to different network conditions. Hybrid routing protocols combine the advantages of both proactive and reactive protocols. Hybrid MANET routing protocols are lightweight, simple and designed to avoid excessive control overhead. These protocols are classified into node-centric and cluster-centric. A network is organized into clusters, or groups, in a cluster-centric network. The clustering optimizes the use of resources and reduces the size of routing tables. Cluster-centric protocols adopt different rules for intercluster data traffic and intracluster traffic. Node-centric protocols are simple protocols that incorporate sender and receiver information. They include fisheye routing protocols, zone routing protocols and a two-zone routing protocol.

\section{A. AODV (AdHoc On-Demand Distance Vector Routing) :}

\section{Protocol USED In ManeT}

It is a reactive routing protocol, meaning that it establishes a route to a destination only on demand. When the valid route is not known by the source node, it initializes a route discovery process by broadcasting a Route Request (RREQ) to its neighbors. Each node discards Route Requests (RREQs) it has already seen by checking the Broadcast ID and the Sequence Number which had been included into the Route Request (RREQ).

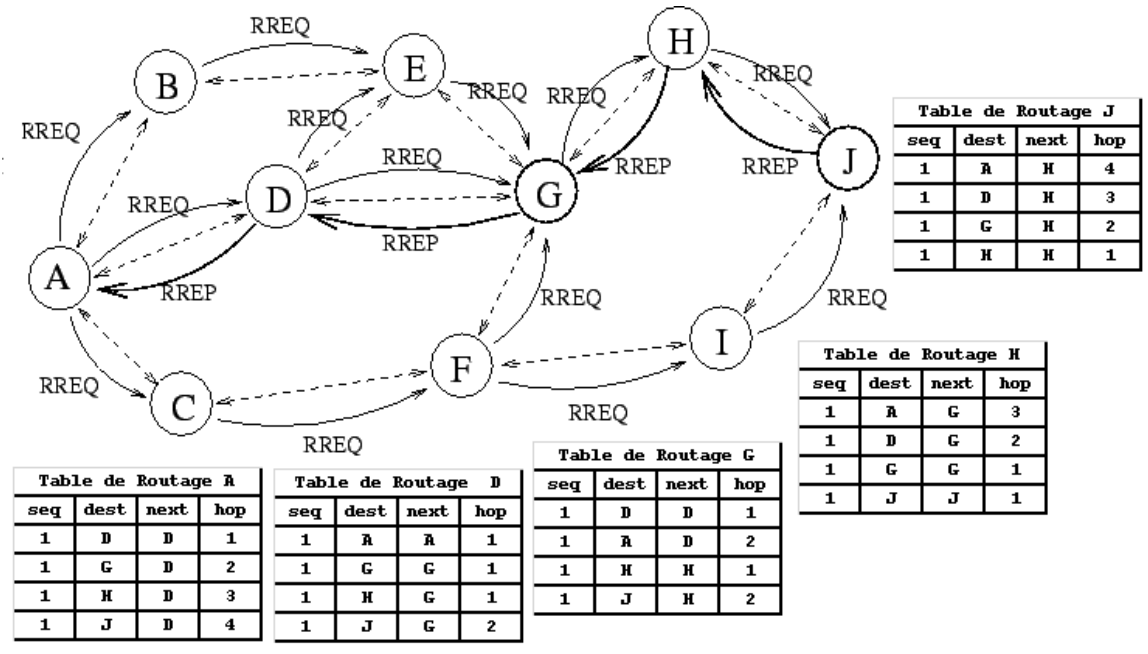

B. DSR (Dynamic Source Routing) :- Determining source routes require accumulating the address of each device between the source and destination during route discovery.

The accumulated path information is cached with nodes processing the route discovery packets. The learned paths are used to route packets. To accomplish source routing, the routed packets contain the address of each device the packet will traverse. protocol features. All

C.Zone Routing Protocol: -Zone routing protocol combines Proactive protocol features and Reactive

Nodes within hop distance at most $\mathrm{d}$ from a node $\mathrm{X}$ are said to be in the routing zone of node $\mathrm{X}$. All nodes at hop distance exactly $\mathrm{d}$ is said zone. In Zone Routing Protocol Intra-zone routing involves maintaining state information for links within a short distance from any given node whereas Inter-zone routing involves using a route discovery protocol for determining routes to far away nodes.

\section{Methodology}

Distance sequence distance vector is a conventional modification of Bellmen-Ford routing algorithm. This protocol adds sequence number, attribute for each route table in each node. A routing table is maintained at each node and this table transmits data packets to other nodes in the network. This protocol was motivated for the use of data exchange along with changing and arbitrary paths of interconnection which may not be close to any base station. All stations list available in the destinations and number of nodes required to transmit data to the destination in the routing table. The routing entry is tagged with the sequence number which is originated by the destination station. For consistency this, each station transmits and updates routing table periodically. The packets being broadcasted between stations indicate which station is sending and number of hops required to reach the particular station. The data broadcast by each node will contain a new sequence number and destination address, the number of hops required to reach the destination and new sequence number, originally stamped by the destination in each new route table 


\section{OUR PROPOSED WORK}

To improve the performance of DSDV algorithm we find out 4 successive steps.

Step 1: The Broken node first launches a port scan attack on the destination node and finds out all the open ports.

Step 2: Then it induces itself into the routing path between the source node and the destination by emitting protocol-compliant messages from leading both Source and Destination nodes to choose such a link for their communications.

Step 3: The Broken node now starts altering the data packet's that are routed through it such that the Destination port number is changed to any other port that is open at the Destination node. The open ports at the Destination have already been found out by a Port Scan Attack.

Step 4: The Broken node will also have to change back the port no of the acknowledgement packets to the original port number, if they are routed through the same link (assume links are bidirectional).

\section{PROPOSED Algorithm}

Algorithm : Modified DSDV Routing Algorithm.

ALGORITHM_STARTS :

I. If the next hop of Source $S$ is not active then use Standard DSDV protocol to transfer a data packet to the Destination $D$.

If (Source.NextHop( $)==$ DOES NOT ACTIVE) then

\{ "Deliver packet from Source S to Destination D by using Standard DSDV." \}

II. If the next hop of Source $S$ is active but the Bufferlength of $S$ is filled up (no space remains available) then discard the Data packet.

Else

If (Source.Buffer_Lngth ()$==$ MAXIMUM ) then

$\{$ "Discard packet" $\}$

III. Alternatively if the capacity of source S is NULL (means bufferlength of Source is zero or emptyindicating any stored data packet would be there) then source can receive but can't send any data packet.

If (Source.Buffer_Lngth $($ ) $==$ ZERO ) then

\{ "Packet will placed inside the Source.Buffer( ) " \}

IV. Now source broadcasts a message to all neighbours by requesting a successive route from source to destination through which a data packet can be delivered.

Source.Brodcasts (ROUTE_REQUEST, 1, Destination D, Source S);

$V$. If the next neighbour of Source $S$ has a route to destination $D$ for packet transferring then $S$ can collect an acknowledgement about Route. Otherwise S already has route acknowledgement packets.

If (Source.NEXT_NEIGHBOUR has route to D) then

\{ Source.RECEIVE (ROUTE_ACK);

$$
\begin{aligned}
& \text { Maximum_no_of_Hops }=0 \text {; } \\
& \text { Minimum_no_of_Hops }=\infty \text {; } \\
& \text { NextHop }=0 ; \\
& \text { Updated_Time }=1 ;
\end{aligned}
$$

VI. When S already has route acknowledgement packets. Then following pseudocode produce the values of Host_port_no, Maximum_no_of_Hops \& Updated_Time.

Else ( Source S has ROUTE_ACK Packets )

\{If (ROUTE_ACK . no_of_Hops $<=$ Max_no_of_Hops)

\{ If (ROUTE_ACK . no_of_Hops $<=$ Max_no_of_Hops) 


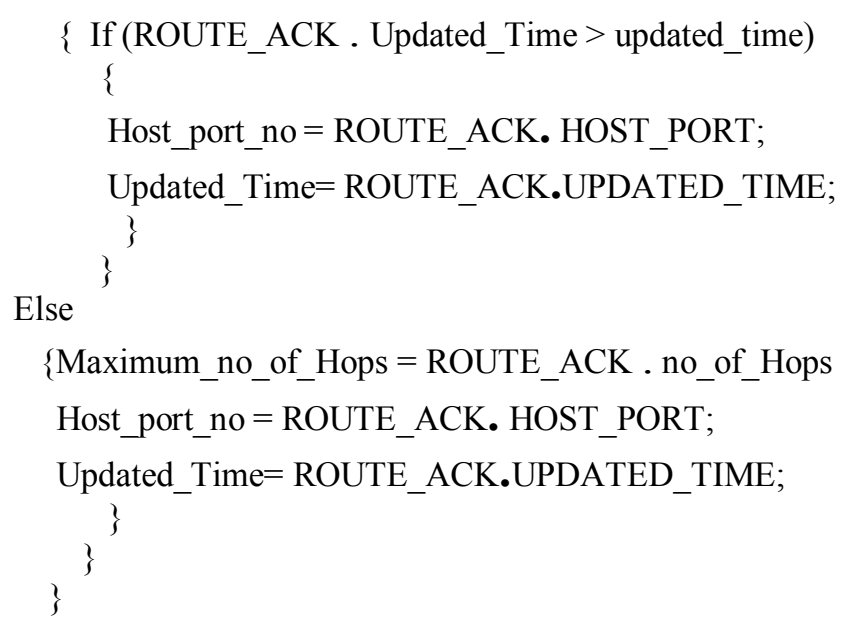

VII. Now Forward the incomming packet via Host_port __no and Updated_Time $=$ ROUTE_ACK.UPDATD_TIME.

\section{ALGORITHM ENDS}

VII. Proposed And Complete PSEudocode

If (Source.NextHop( $)==$ DOES NOT ACTIVE) then

\{ "Deliver packet from Source S to Destination D by using Standard DSDV." ; \} Else

If (Source.Buffer_Lngth $($ ) $==$ MAXIMUM ) then

$\{$ "Discard packet ";

If (Source.Buffer_Lngth ()$==$ ZERO $)$ then

\{ "Packet will placed inside the Source.Buffer( ) "; \}

Source.Brodcasts (ROUTE_REQUEST, 1, Destination D, Source S);

If (Source.NEXT_NEIGHBOUR has route to D) then

\{ Source.RECEIVE (ROUTE_ACK);

Maximum_no_of_Hops $=0$;

Minimum_no_of_Hops $=\infty$;

NextHop $=0$;

Updated_Time $=1$;

Else ( Source S has ROUTE_ACK Packets )

\{If (ROUTE_ACK. no_of_Hops $<=$ Max_no_of_Hops)

\{If (ROUTE_ACK . no_of_Hops $<=$ Max_no_of_Hops)

\{If (ROUTE_ACK. Updated_Time $>$ updated_time)

$\left\{\right.$ Host $\_$port_no $=$ROUTE_ACK. HOST_PORT;

Updated_Time $=$ ROUTE_ACK.UPDATED_TIME;

\}

\}

Else

$\{$ Maximum_no_of_Hops $=$ ROUTE_ACK . no_of_Hops

Host_port_no $=$ ROUTE_ACK. HOST_PORT;

Updated_Time $=$ ROUTE_ACK.UPDATED_TIME; 


\section{Performance ANAlysis OF MOdified DSDV By USING NS2 Simulator}

We use NS2 to simulate our proposed algorithm. A simulation study was carried out to evaluate the performance of MANET routing protocols such as DSDV, and Improved-DSDV based on the metrics throughput, packet delivery ratio and average end-to-end delay with the following parameters:

List of Simulation parameters:

Simulator: - NS-2

Node Movement: - Random

Max Speed :- $0-28 \mathrm{~m} / \mathrm{s}$

Traffic Type :- UDP

Bandwidth :- $1 \mathrm{Mb} / \mathrm{s}$

No of Nodes :- 30

While comparing two protocols, we focused on two performance measurements such as Average Delay, Packet Delivery Fraction

\section{A. Packet delivery Ratio:-}

Packet Delivery Ratio (PDR) is the ratio between the number of packets transmitted by a traffic source and the number of packets received by a traffic sink. It measures the loss rate as seen by transport protocols and as such, it characterizes both the correctness and efficiency of ad hoc routing protocols. A high packet delivery ratio is desired in any network.

\section{B. Average End-to-End delay:-}

The packet End-to-End delay is the average time that a packet takes to traverse the network. This is the time for generation of the packet in the sender upto it's reception at the destinations application layer and it is measured in seconds. It therefore includes all the delays in the network such as buffer queues, the transmission time and delays induced by routing activities and MAC control exchanges. Control overhead: The control overhead is defined as the total number of routing control packets normalized by the total number of receiving data packets.

\section{PERFORMANCE COMPARISON BETWEen STANDARD DSDV AND OUR MODIFIED DSDV}

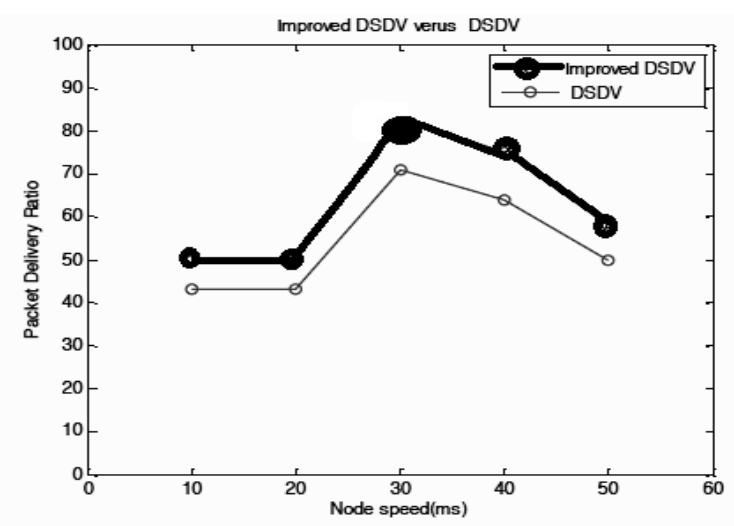

Fig. Packet Delivery Ratio Vs Node Speed (ms)

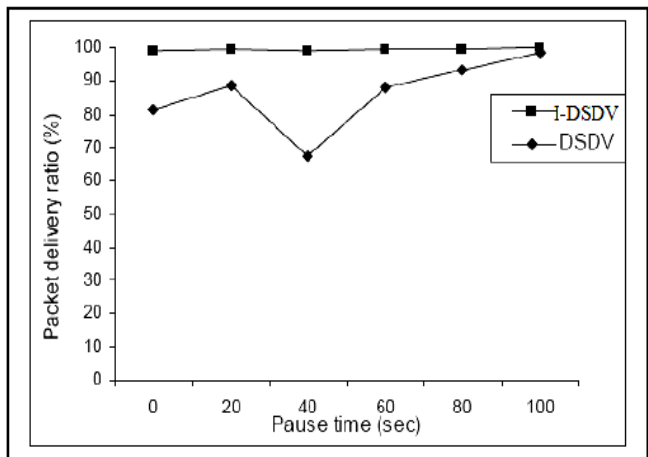

Fig. Packet delivery ratio vs pause time for 50 nodes with 30 sources 


\section{Conclusion}

Improved -DSDV overcomes the problem of state routes, and thereby improves the performance of regular DSDV. Simulation results show that both of the protocols deliver a greater percentage of the originated data packets when there is little node mobility, converging to $100 \%$ delivery ratio when there is no node motion. In current state problem of DSDV is routing information will maintain at each node locally in the network. All routing decisions are taken completely in distributed fashion. So the local information may be old and invalid, local information updates periodically. We compare the performance of our work with DSDV and improved DSDV. In our improved DSDV routing protocol, nodes can cooperate together to Obtain an objective opinion about another nodes trustworthiness. They can also perform trusted routing behaviors according to the trust relationship among them.

\section{Book:}

\section{REFERENCES}

[1] J. Clerk Maxwell, A Treatise on Electricity and Magnetism, 3rd ed., vol. 2. Oxford: Clarendon, 1892, pp.68-73.

[2] M. Young, The Technical Writer's Handbook. Mill Valley, CA: University Science, 1989.

\section{Journal Paper:}

[3] G. Eason, B. Noble, and I. N. Sneddon, "On certain integrals of Lipschitz-Hankel type involving products of Bessel functions," Phil. Trans. Roy. Soc. London, vol. A247, pp. 529-551, April 1955. (references)

[4] I. S. Jacobs and C. P. Bean, "Fine particles, thin films and exchange anisotropy," in Magnetism, vol. III, G. T. Rado and H. Suhl, Eds. New York: Academic, 1963, pp. 271-350.

\section{Proceeding Papers:}

[5] K. Elissa, "Title of paper if known," unpublished.

[6] R. Nicole, "Title of paper with only first word capitalized," J. Name Stand. Abbrev., in press.

[7] Y. Yorozu, M. Hirano, K. Oka, and Y. Tagawa, "Electron spectroscopy studies on magneto-optical media and plastic substrate interface," IEEE Transl. J. Magn. Japan, vol. 2, pp. 740-741, August 1987 [Digests 9th Annual Conf. Magnetics Japan, p. 301, 1982]. 